\title{
The Zika Virus Transmission Model
}

\section{Jiraporn Lamwong ${ }^{*}$, Puntani Pongsumpun ${ }^{2}$}

1 Department of General Basis, Thatphanom College, Nakhon Phanom University, Thailand.

2 Department of Mathematics, Faculty of Science, King Mongkut's Institute of Technology Ladkrabang, Thailand.

* Corresponding author. Email: kppuntan@kmitl.ac.th Manuscript submitted October 12, 2016; accepted January 12, 2017. doi: 10.17706/ijbbb.2017.7.2.66-73

\begin{abstract}
A mathematical model of Zika virus is studied in this paper. Zika is caused by Zika virus, a flavivirus related to yellow fever, dengue. In 1952, first outbreak occurred in Uganda. In 1962, an epidemic was recognized as the first time in Thailand. In this study, we consider the transmission cycle between two population groups: human and mosquito. Using standard dynamical modeling method, the stability conditions of our model is considered by Routh-Hurwitz criteria. The numerical simulations are shown to support the analytical results.
\end{abstract}

Key words: Basic reproductive number, disease free steady state, endemic steady state, stability, Zika.

\section{Introduction}

On 6 July 2016, World Health Organization (since 2007) reported evidence of zika virus from 65 countries [1]. There are 61 countries reported mosquito-borne zika virus transmission. Guinea-Bissau is the last country where there is the report of mosquito-borne zika virus [1], [2]. Zika virus is caused by a virus transmitted by Aedes mosquitoes, Family flavivirus, related to yellow fever, dengue [3]. In 1947, Zika virus is initially found in monkey of Uganda. In 1952, Zika virus was found in human [4]-[7].

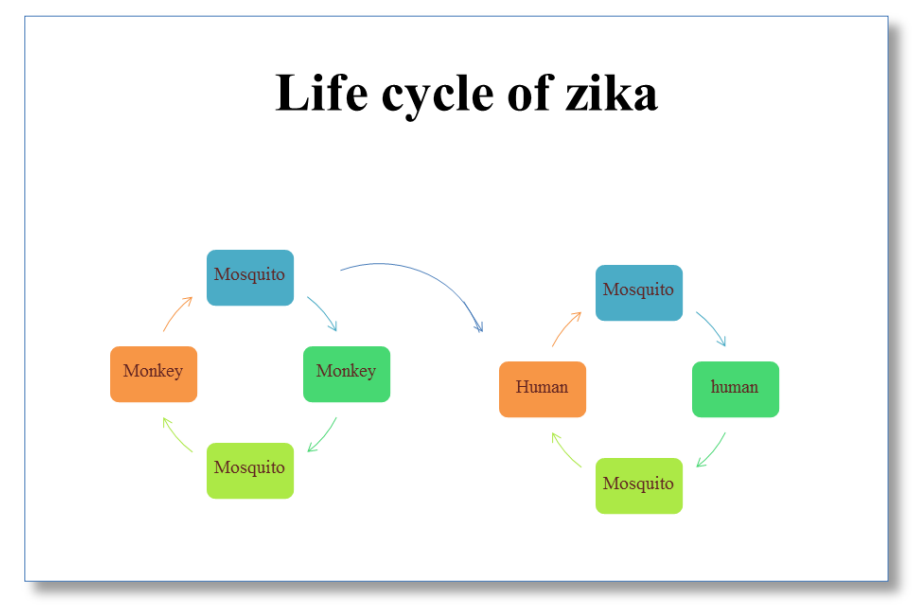

Fig. 1. Life cycle of zika [7]-[9].

In 1952, an epidemic was recognized as the first time in Thailand. The first case is the traveler, a female from Canadian. Between 21 January to 4 February, Thailand confirmed zika virus disease in a traveler. 
Human can be infected by mosquito-borne from the Aedes genus. This is the same mosquito that transmits dengue, chikungunya and yellow fever [10]. In 2016, Gao et al. [4], studied mathematical model of zika virus as mosquito-borne disease. They considered sexual transmission, biting rate and mortality rate of mosquito. They used sensitivity analysis to analyze the basic reproductive number $\left(\mathrm{R}_{0}\right)$. Kucharski et al. [11] use a mathematical model of vector-borne infections, the transmission dynamical of zika virus in island population and examined the transmission dynamical of zika virus on six archipelagos in Frence in 2013-14. Bichare et al. [12] identified the impact of short term mobility between two idealized interconnected communities, using a Lagrangina mode land show simulations of short term mobility. In 2011, Naowawat, et al. [13], studied dynamical model for determining human susceptible to dengue fever. The basic reproductive number is explained as follows: $\mathrm{R}_{0}<1$, the disease-free state is local stable. The endemic state is local stable for $\mathrm{R}_{0}>1$. In this paper, we consider the transmission of zika virus. We divided the human population into susceptible, exposed, infectious and recovered classes (SEIR models). Mosquito population is separated into susceptible, exposed and infectious classes (SEI model). Using standard dynamical modeling method, the stability of model is determined by using Routh-Hurwitzcriteria. The Threshold parameter is found to separate the different behaviors of two steady states. The numerical simulations are shown to support the analytical results.

\section{Mathematical Model}

In our model, we consider the transmission of zika virus between two population, human population and mosquito population. The human population is divided into four sub-groups: susceptible, exposed, infected and recovered. The mosquito is divided into three sub-groups: susceptible, exposed and infected. The variables and parameters in our equations are described as follows:

$S_{h}$ is the size of susceptible human populations,

$E_{h}$ is the size of exposed human population,

$I_{h}$ is the size of infected human population,

$R_{h}$ is the size of recovered human population,

$S_{v}$ is the size of susceptible mosquito population,

$E_{v}$ is the size of exposed mosquito population,

$I_{v}$ is the size of infected mosquito population,

$B$ is the birth rate of human population,

$d_{v}$ is the natural death rate of human populations,

$\varepsilon$ is the biting rate of mosquito population,

$\beta_{h}$ is the rate at which mosquito to human Zika is contracted,

$F_{h}$ is the incubation period of Zika virus in human,

$\gamma$ is the recovery rate of human populations,

$N_{h}$ is the number of human populations,

$m$ is the number of other animals that the mosquito can feed on

$A$ is the recruitment rate of mosquitoes

$d_{v}$ is the natural death rate of mosquito

$\beta_{v}$ is the rate at which human to mosquito Zika is contracted,

$F_{v}$ is the incubation period of Zika virus in mosquito,

The diagram of our dynamical systems can be described by following Fig. 2:

The dynamical rate of each population group is described by following equations. 
For human:

$$
\begin{gathered}
\frac{d S_{h}}{d t}=B N_{h}-\frac{\varepsilon \beta_{h} S_{h} I_{v}}{N_{h}+m}-d_{h} S_{h} \\
\frac{d E_{h}}{d t}=\frac{\varepsilon \beta_{h} S_{h} I_{v}}{N_{h}+m}-\left(F_{h}+d_{h}\right) E_{h} \\
\frac{d I_{h}}{d t}=F_{h} E_{h}-\left(\gamma+d_{h}\right) I_{h} \\
\frac{d R_{h}}{d t}=\gamma I_{h}-d_{h} R_{h}
\end{gathered}
$$

For mosquitoes:

$$
\begin{gathered}
\frac{d S_{v}}{d t}=A-\frac{\varepsilon \beta_{v} S_{v} I_{h}}{N_{h}+m}-d_{v} S_{v} \\
\frac{d E_{v}}{d t}=\frac{\varepsilon \beta_{v} S_{v} I_{h}}{N_{h}+m}-\left(F_{v}+d_{v}\right) E_{v} \\
\frac{d I_{v}}{d t}=F_{v} E_{v}-d_{v} I_{v}
\end{gathered}
$$

where $N_{h}=S_{h}+E_{h}+I_{h}+R_{h}$ and $N_{v}=S_{v}+E_{v}+I_{v}$

We can be normalize our equations (1)-(7) by introducing the new variables:

$$
s_{h}=\frac{S_{h}}{N_{h}}, e_{h}=\frac{E_{h}}{N_{h}}, i_{h}=\frac{R_{h}}{N_{h}}, r_{h}=\frac{R_{h}}{N_{h}}, s_{v}=\frac{S_{v}}{N_{v}}=\frac{S_{v}}{\left(A / d_{v}\right)}, e_{v}=\frac{E_{v}}{N_{v}}=\frac{E_{v}}{\left(A / d_{v}\right)} \text { and } i_{v}=\frac{I_{v}}{N_{v}}=\frac{I_{v}}{\left(A / d_{v}\right)}
$$

Then we have the dynamical equations of the reduced equations as follows:

$$
\begin{aligned}
\frac{d s_{h}}{d t} & =B-\frac{\varepsilon \beta_{h} A s_{h} i_{v}}{d_{v}\left(N_{h}+m\right)}-d_{h} s_{h} \\
\frac{d e_{h}}{d t} & =\frac{\varepsilon \beta_{h} A s_{h} i_{v}}{d_{v}\left(N_{h}+m\right)}-\left(F_{h}+d_{h}\right) e_{h} \\
\frac{d i_{h}}{d t} & =F_{h} e_{h}-\left(\gamma+d_{h}\right) i_{h} \\
\frac{d s_{v}}{d t} & =d_{v}-\frac{\varepsilon \beta_{v} N_{h} s_{v} i_{h}}{N_{h}+m}-d_{v} s_{v} \\
\frac{d i_{v}}{d t} & =F_{v}\left(1-i_{v}-s_{v}\right)-d_{v} i_{v}
\end{aligned}
$$

with the condition $s_{h}+e_{h}+i_{h}+r_{h}=1$ and $s_{v}+e_{v}+i_{v}=1$

\section{Analysis of Mathematical Model}

\subsection{Analytical Solutions}

After we formulate the dynamical equations, the next step is to find the steady states of our equations. The standard dynamical modeling method is used for analysis of our model, a disease free steady state and 
an endemic steady state are obtained. We set the right hand side of equations (8) to zero, then we obtain the steady states:

i) Disease free steady state: $\bar{E}_{0}=\left(s_{h}^{*}, e_{h}^{*}, i_{h}^{*}, s_{v}^{*}, i_{v}^{*}\right)=(1,0,0,1,0)$

ii) Endemic steady state: $\bar{E}_{1}=\left(s_{h}^{*}, e_{h}^{*}, i_{h}^{*}, s_{v}^{*}, i_{v}^{*}\right)$ where

$$
\begin{gathered}
s_{h}^{*}=\frac{B d_{v}\left(N_{h}+m\right)}{d_{h} d_{v}\left(N_{h}+m\right)+\frac{A F_{v} N_{h} \beta_{h} \beta_{v} \varepsilon^{2} i_{h}^{*}}{\left(d_{v}+F_{v}\right)\left(d_{v}\left(N_{h}+m\right)+\varepsilon N_{h} \beta_{v} i_{h}^{*}\right)}} \\
e_{h}^{*}=\frac{A B F_{v} N_{h} \beta_{h} \beta_{v} \varepsilon^{2} i_{h}^{*}}{\left(d_{h}+F_{h}\right)\left(A F_{v} N_{h} \beta_{h} \beta_{v} \varepsilon^{2} i_{h}^{*}+d_{h} d_{v}\left(d_{v}+F_{v}\right)\left(N_{h}+m\right)\left(d_{v}\left(N_{h}+m\right)+N_{h} \beta_{v} \varepsilon i_{h}^{*}\right)\right)} \\
i_{h}^{*}=-\frac{d_{h} d_{v}^{2}\left(d_{h}+F_{h}\right)\left(d_{v}+F_{v}\right)\left(N_{h}+m\right)^{2}\left(d_{h}+\gamma\right)-A B F_{h} F_{v} N_{h} \beta_{h} \beta_{v} \varepsilon^{2}}{\left(d_{h}+F_{h}\right) N_{h} \beta_{v}\left(d_{h}+\gamma\right) \epsilon\left(d_{h} d_{v}\left(d_{v}+F_{v}\right)\left(N_{h}+m\right)+A F_{v} \beta_{h} \varepsilon\right)} \\
s_{v}^{*}=\frac{d_{v}\left(N_{h}+m\right)}{d_{v}\left(N_{h}+m\right)+N_{h} \beta_{v} \varepsilon i_{h}^{*}} \\
i_{v}^{*}=\frac{F_{v} N_{h} \beta_{v} \varepsilon i_{h}^{* *}}{\left(d_{v}+F_{v}\right)\left(d_{v}\left(N_{h}+m\right)+N_{h} \beta_{v} \varepsilon i_{h}^{*}\right)}
\end{gathered}
$$

By using standard dynamic modeling method, the local stability of each steady state is defined of all eigenvalues. If all eigenvalues for each steady state produce the negative real part, we can conclude that steady state is local stability [14]. The eigenvalues are the solutions of the characteristic equation

$$
\left|J_{\bar{E}_{i}}-\lambda I_{5}\right|=0, i=0,1
$$

where $J_{\bar{E}_{i}}$ is the Jacobian matrix; $i=0,1$ and $\mathrm{I}$ is the identity matrix.

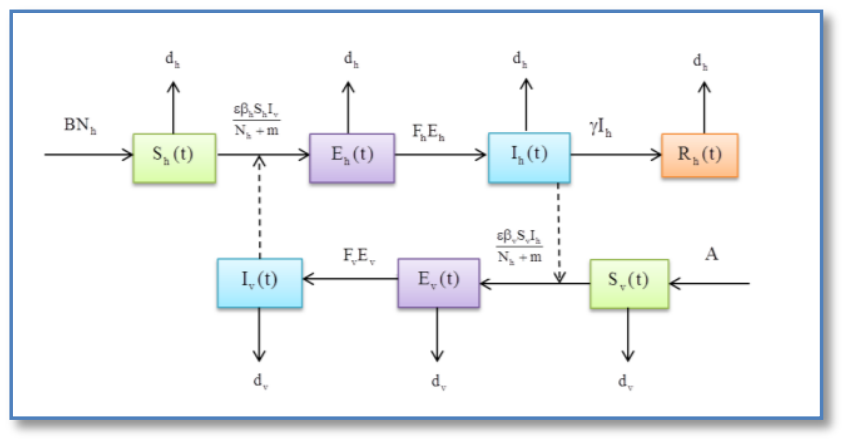

Fig.2 flowchart of the model.

Disease free steady state, the characteristic equation is $\bar{E}_{0}=\left(s_{h}^{*}, e_{h}^{*}, i_{h}^{*}, s_{v}^{*}, i_{v}^{*}\right)=(1,0,0,1,0)$

$$
\left|\begin{array}{ccccc}
-d_{h}-\lambda & 0 & 0 & 0 & -\frac{\varepsilon A \beta_{h}}{d_{v}\left(N_{h}+m\right)} \\
0 & -\left(d_{h}+F_{h}\right)-\lambda & 0 & 0 & \frac{\varepsilon A \beta_{h}}{d_{v}\left(N_{h}+m\right)} \\
0 & F_{h} & -\left(d_{h}+\gamma\right)-\lambda & 0 & 0 \\
0 & 0 & -\frac{\varepsilon \beta_{v} N_{h}}{N_{h}+m} & -d_{v}-\lambda & 0 \\
0 & 0 & 0 & -F_{v} & \left(-d_{v}-F_{v}\right)-\lambda
\end{array}\right|=0
$$


The characteristic equation of the above Jacobian matrix is

$$
\left(-d_{h}-\lambda\right)\left(\lambda^{4}+A_{1} \lambda^{3}+A_{2} \lambda^{2}+A_{3} \lambda+A_{4}\right)=0
$$

We can see all eigenvalues have negative real parts. We use Routh-Hurwitz criteria [15]. Where $R_{0}<1$

$$
R_{0}=\frac{F_{h} F_{v} \beta_{h} \beta_{v} N_{h} \varepsilon^{2}}{d_{v}^{2}\left(d_{h}+F_{h}\right)\left(d_{v}+F_{v}\right)\left(N_{h}+m\right)^{2}\left(d_{h}+\gamma\right)}
$$

Therefore, we can conclude that the endemic steady state is local stability for $\mathrm{R}_{0}>1$ and the disease free steady state is local stability $R_{0}<1$.

\subsection{Numerical Solutions}

The values of parameter in the model are shown in Table 1.

\begin{tabular}{|c|c|c|}
\hline parameter & Values & Source \\
\hline$\varepsilon$ & 0.5 & {$[4,10]$} \\
\hline$\beta_{v}$ & 0.05 & {$[4,10]$} \\
\hline$F_{v}$ & $1 / 5$ & {$[4,7,10]$} \\
\hline$d_{v}$ & $1 / 14$ & {$[4,7,10]$} \\
\hline$F_{h}$ & $1 / 5$ & {$[6]$} \\
\hline$\gamma$ & $1 / 20$ & [11] \\
\hline$B$ & $1 /(65 \times 365)$ & [16] \\
\hline$d_{h}$ & $1 /(75 \times 365)$ & [16] \\
\hline$\beta_{h}$ & 0.4 & Assumed \\
\hline$N_{h}$ & 1 & Assumed \\
\hline$m$ & 1 & Assumed \\
\hline$A$ & 10 & Assumed \\
\hline \multicolumn{2}{|c|}{ Disease free } & \\
\hline \multicolumn{2}{|c|}{ Endemic } & $\mathrm{R}_{0}=2.8557$ \\
\hline
\end{tabular}

Table 1. Values of Parameter

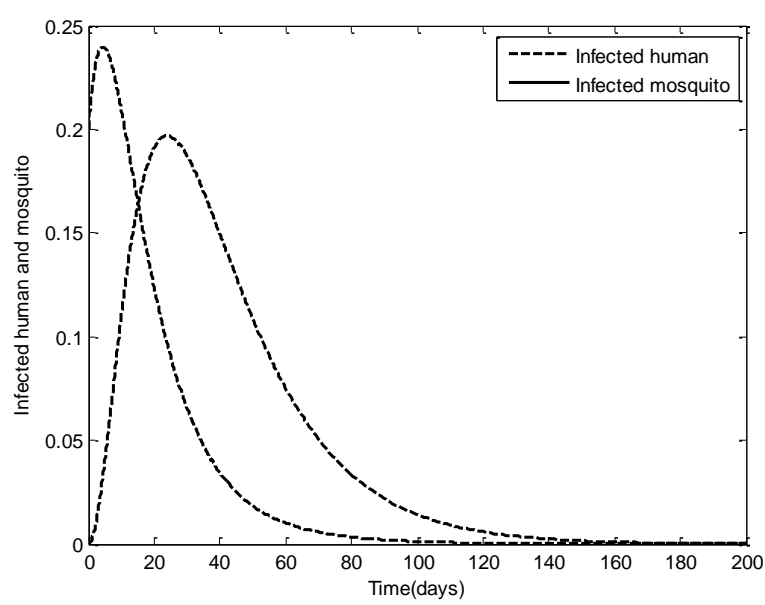

Fig. 3. Time series solution of infected human and infected mosquito. 

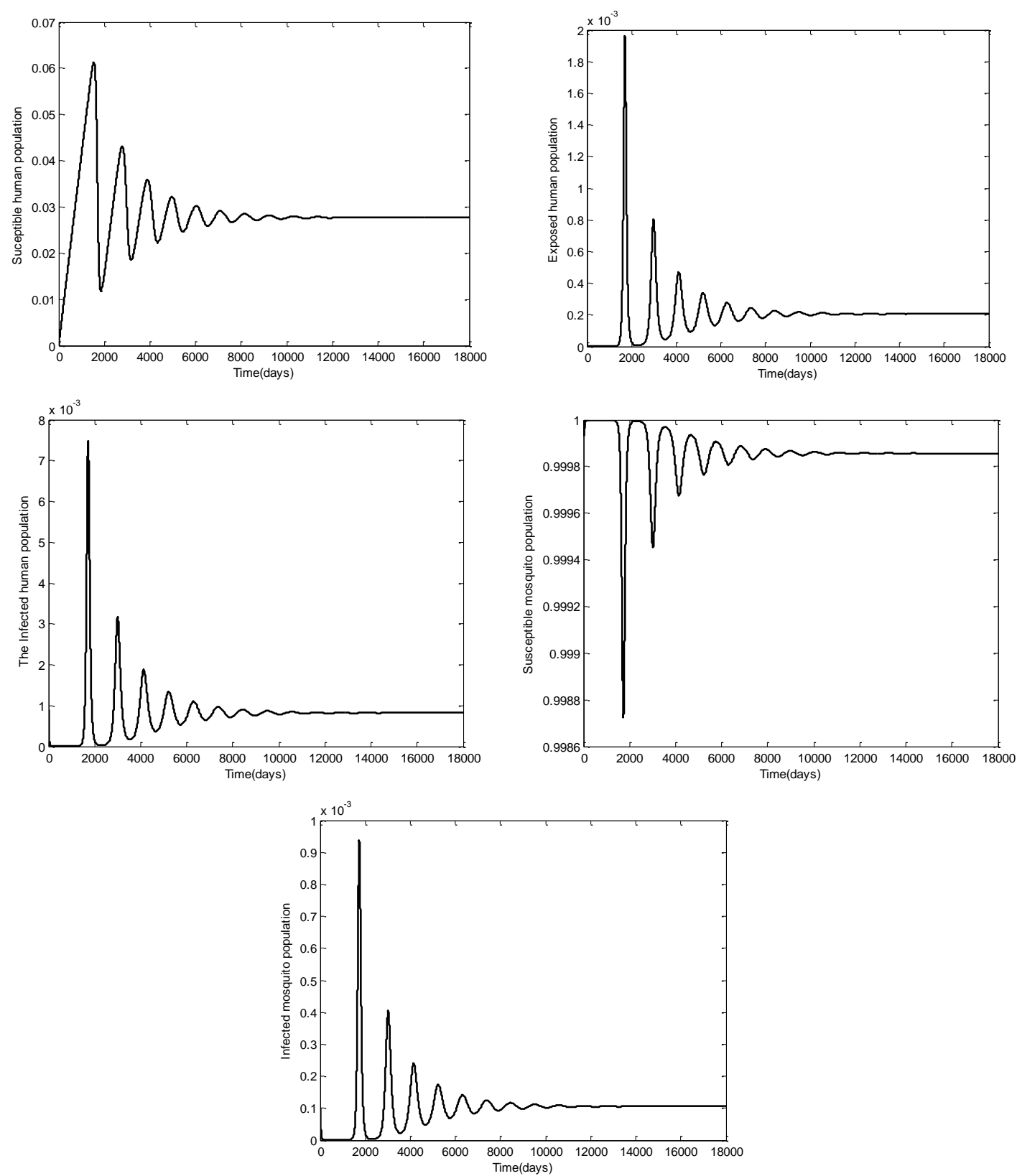

Fig. 4. Time series solution of susceptible human, exposed human, infected human, susceptible mosquito and infected mosquito on endemic steady state, the solutions converge to $(0.0277,0.0002,0.0008,0.9999,0.0001)$ where $R_{0}=2.8557$.

\section{Conclusions}

In this study, we constructed the dynamical transmission model of Zika virus. The model has two steady state, disease free steady and endemic steady state. The basic reproductive number [16], [17] is denoted by $R_{0}$ where

$$
R_{0}=\frac{F_{h} F_{v} \beta_{h} \beta_{v} N_{h} \varepsilon^{2}}{d_{v}^{2}\left(d_{h}+F_{h}\right)\left(d_{v}+F_{v}\right)\left(N_{h}+m\right)^{2}\left(d_{h}+\gamma\right)}
$$

Fig. 2 is time series solution of infected human and infected mosquito, where $R_{0}<1$. And Fig. 3 is time series solution of susceptible human, exposed human, infected human, susceptible mosquito and infected 
mosquito on endemic steady state, the solutions converge to $(0.0277,0.0002,0.0008,0.9999,0.0001)$ where $R_{0}=2.8557$.

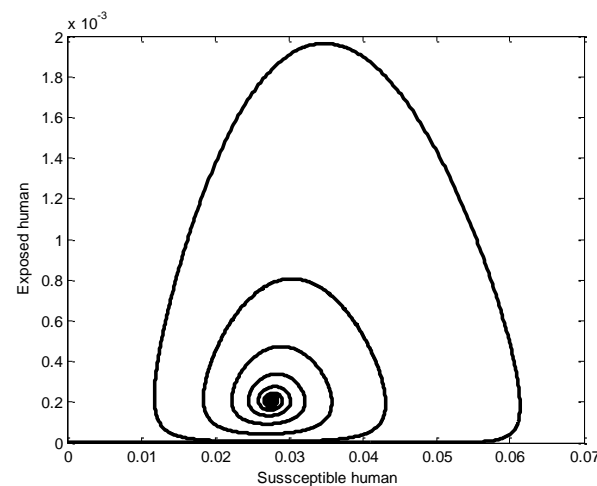

(a)

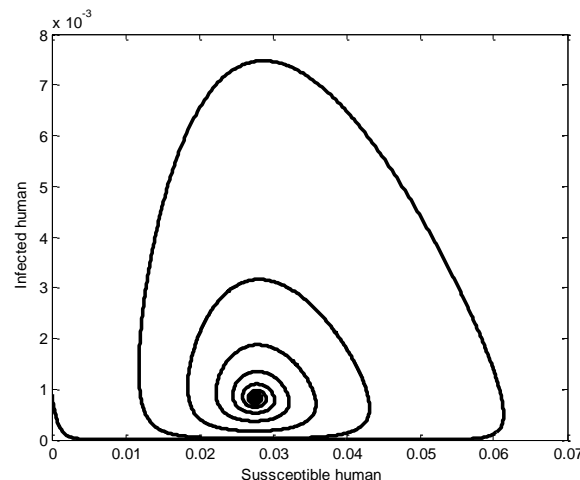

(b)

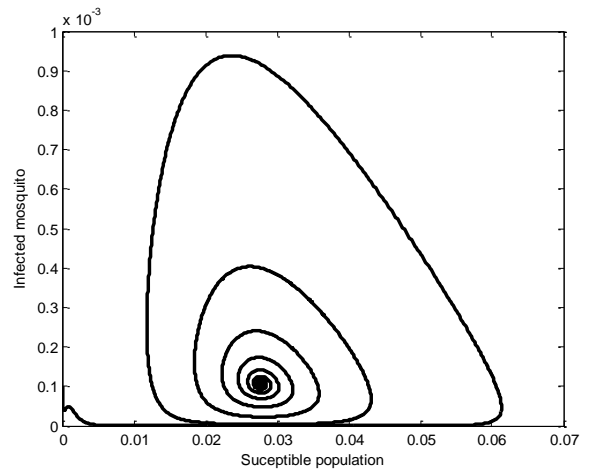

(c)

Fig. 5. Numerical solution of our dynamical equations. where $R_{0}>1$.

\section{Acknowledgment}

This work is supported by Thatphanom College, Nakhon Phanom University and Faculty of Science, King Mongkut's Institute of Technology Ladkrabang, Thailand.

\section{References}

[1] World Health Organization. Zika virus situation reports. Retrieved from the website: http://www.who.int/emergencies/zika- virus/situation-report/7-july-2016/en/

[2] Ministry of Public Health. Zika virus disease. Retrieved from the website: http://beid.ddc.moph.go.th/beid_2014/_node/2078

[3] Victor, M. Role of short dispersal on the dynamics of Zika virus.

[4] Gao, D. Z., et al. (2016). Prevention and control of Zika as a mosquito-borne and sexually transmitted disease: A mathematical modeling analysis.

[5] Dick, G. W., Kitchen, S. F., \& Haddow, A. J. (1952). Zika virus. I. Isolations and serological specificity. Trans. R. Soc. Trop. Med. Hyg., 46(5), 509-520.

[6] Macnamara, F. N. (1954). Zika virus: A report on three cases of human infection during an epidemic of jaundice in Nigeria. Trans. R. Soc. Trop. Med. Hyg., 48(2), 139-145.

[7] Boorman, J. P., \& Porterfield, J. S. (1956). A simple technique for infection of mosquitoes with viruses transmission of Zika virus. Trans. R. Soc. Trop. Med. Hyg., 50(3), 238-242

[8] Zika virus net. Zika virus transmission. Retrieved from the website: 
http://www.zikavirusnet.com/transmission.html

[9] Ministry of Public Health. Zika virus disease. Retrieved from the website: http://beid.ddc.moph.go.th/beid_2014/node/ 2078

[10] Andraud, M., Hens, N., Marais, C. \& Beutels, P. (2012). Dynamic epidemiological models for dengue transmission: a systematic review of structural approaches. PLoS One, 7(11), e49085.

[11] Adam, J. K., Sebastian, F., Rosalind, M. E., Henri-Pierre, M., Edmunds, W. J., \& Eric, J. N. (2016). Transmission dynamics of Zika virus in island population: A modeling analysis of the 2013-14. Frenah Polynesia Outbreak, 1-15, 2016.

[12] Victor, M., Baltazar, E., Derdei, B., Susan, A. H., \& Castillo, C. (2016). Role of short-term dispersal on the dynamics of Zikavirus.

[13] Naowarat, S., Korkistsakul, T., \& Tang, I. M. (2011). Dynamical model for determining human susceptible to dengue fever. 1101-1106.

[14] Leah, E. K. (1988). Mathematical Models in Biology. Random House.

[15] Leah, E.-K. (1988). Mathematical Models in Biology. Random House of United States Publishers.

[16] Official statistics Thailand. Retrieved from the website: http://www. nso. go.th/

[17] Sungchasit, R., Pongsumpun, P., \& Tang, I. M. (2017). Environmental impact on the spread of dengue virus when two mosquito species circulate. Far East Journal of Mathematical Sciences, 101(1), 137-170.

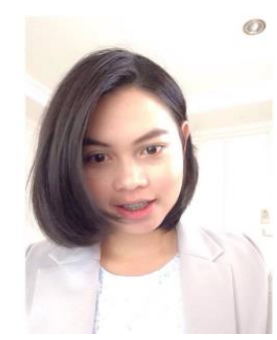

J. Lamwong received her bachelor of science in applied mathematics and the master of science in applied mathematics from King Mongkut's Institute of Technology Ladkrabang, Thailand. From 2015 till date, she is a lecturer of mathematics in Thatphanom College, Nakhon Phanom University. Her research interest is mathematical modelling.

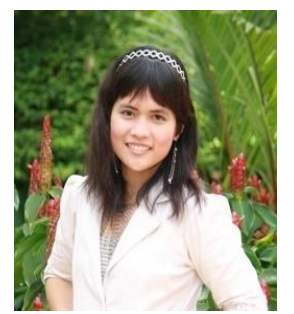

P. Pongsumpun received her bachelor of science in mathematics (second class honors) and the doctor of philosophy in mathematics (international programme) from Mahidol University, Thailand. From 2004 to 2012, she was an as assistant professor of mathematics. From 2012 till date, she is an associate professor of mathematics, thesis Ph.D. and M.Sc. advisors in King Mongkut's Institute of Technology Ladkrabang, Thailand Her research interests are mathematical modelling in medical science, differential equations and numerical analysis. 